\title{
A Manufacturing Collaboratory Case Study - Companion Document
}

Michelle P. Steves Amy J. Knutilla Elizabeth E. Wierba

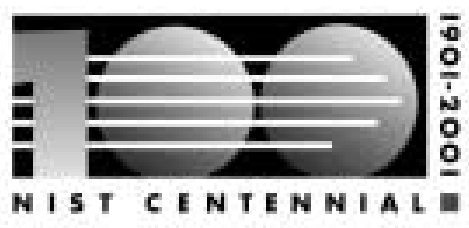

National Institute of Standards and Technology Technology Administration, U.S. Department of Commerce 


\title{
A Manufacturing Collaboratory Case Study - Companion Document
}

\author{
Michelle P. Steves \\ Manufacturing Systems Integration Division \\ Manufacturing Engineering Laboratory \\ Amy J. Knutilla \\ Knutilla Technologies \\ Elizabeth E. Wierba \\ Collaboratory for Research on Electronic Work \\ School of Information \\ University of Michigan
}

July 2001

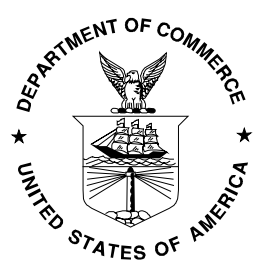

U.S. Department of Commerce Donald L. Evans, Secretary

National Institute of Standards and Technology Karen H. Brown, Acting Director 


\section{Table of Contents}

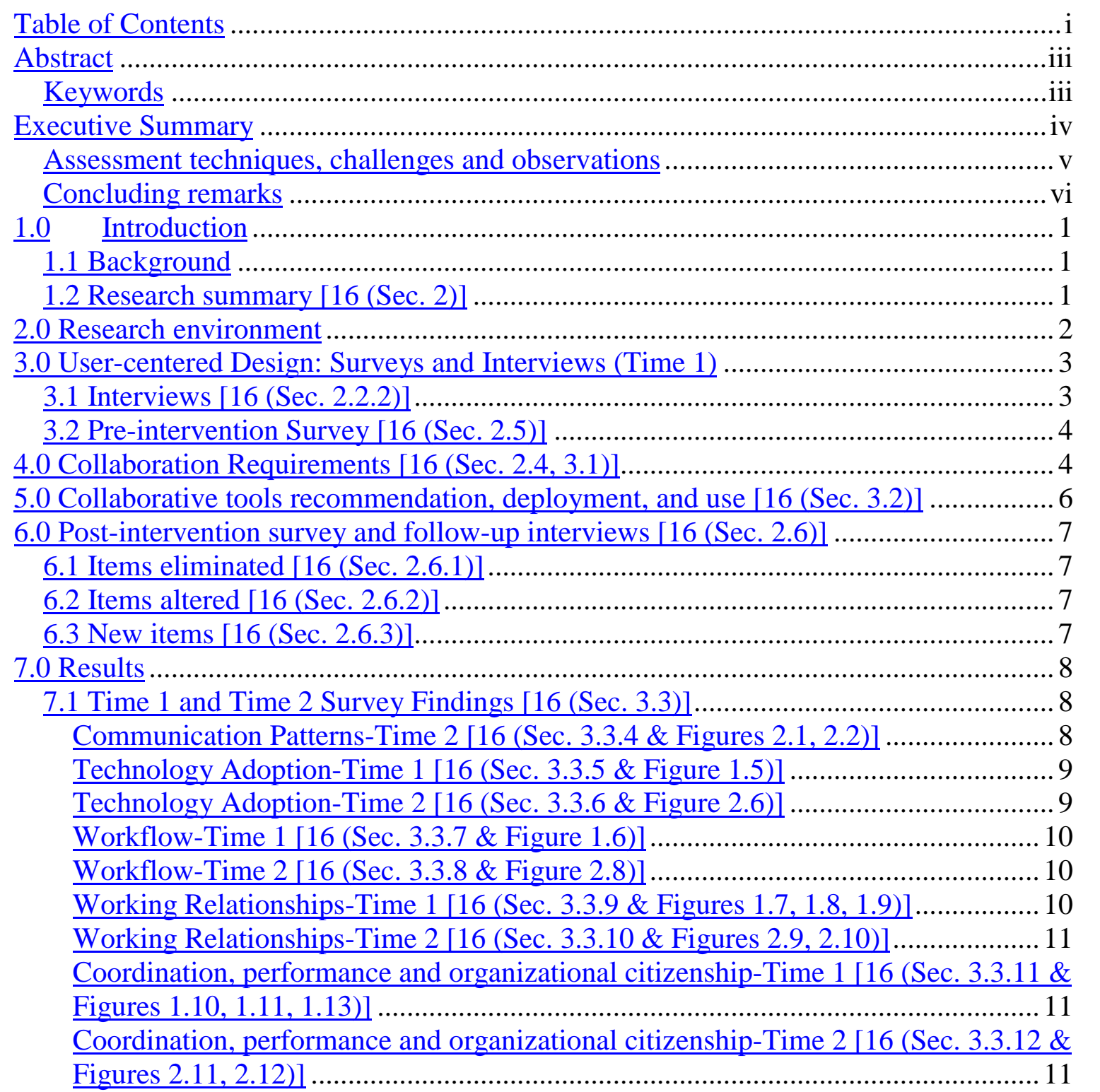

7.2 Comparing Means at Time 1 and Time 2 [16 (Sec. 3.4 \& Appendix I - Tables 8,

9)]

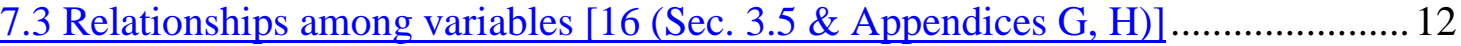

Impact of collaboration tools [16 (Sec. 3.5.1)] ............................................... 12

Performance $[16$ (Sec. 3.5.3)] ........................................................................... 13

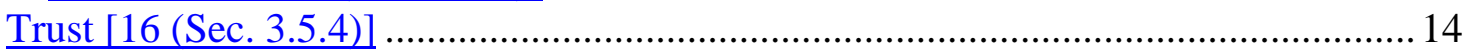

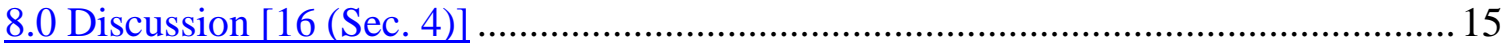

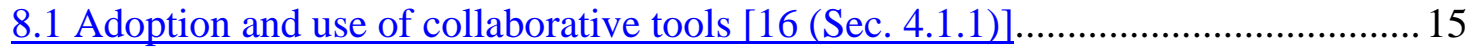

8.2 Impact of collaborative tools [16 (Sec. 4.1.2)].............................................. 16

8.3 Assessment techniques, challenges and observations [16 (Sec. 4.2)] .................. 17

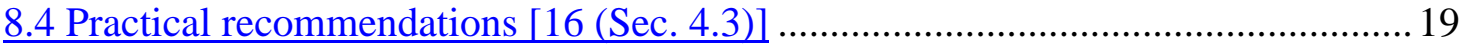


8.4.1 Advice to managers and engineers $[16$ (Sec. 4.3.2)]....................................... 19

8.4.2 Advice to researchers [16 (Sec. 4.3.3)] ....................................................... 21

9.0 Concluding remarks .............................................................................21

References ....................................................................................................21 


\section{Abstract}

This report summarizes a case study of a collaborative tool intervention within a geographically-distributed, product-development team of an automotive parts supplier. This report serves as a companion document to a larger, more detailed volume, prepared as the final report to the National Institute of Standards and Technology (NIST) by the University of Michigan (UM), A Manufacturing Collaboratory Case Study [16]. In this research, an application sharing tool, NetMeeting, was deployed after an in-depth analysis of the team's collaboration requirements. Team members, who were perceived as early technology adopters, were trained in the tool use. Using pre- and postintervention surveys and interviews, UM researchers examined levels of adoption, use of the collaborative tool, and the relationship between tool use and changes in team performance. Results indicated that a small proportion (1/3) of the team used NetMeeting, and that NetMeeting users described a positive impact of this tool on their collaborative work. Lower than expected adoption rates may have been due to key features of NetMeeting, such as synchronous application sharing, not being as valuable as expected for collaborations that spanned time zones. These features were more widely used for local collaborations. Furthermore, workarounds already in use within the team, such as transmitting engineering drawings as email attachments, may have been sufficient to inhibit exploration and adoption of alternative collaboration tools - even when the newly introduced tools had desirable features as described by the users. Results are discussed in terms of the adoption, use, impact of collaboration tools, techniques for assessment and evaluation of collaboration tools, and practical recommendations for users. While the data indicated team performance was mixed, there is an abundance of information useful to researchers and users of tools for collaboration. This report provides the interested reader with an overview of the research, as well as, pointers into the full report [16] for complete detail.

\section{Keywords}

NetMeeting; CSCW; collaboration technologies; collaborative tool adoption 


\section{Executive Summary}

The National Institute of Standards and Technology (NIST) and the University of Michigan (UM) embarked on a research project to examine the deployment of collaboration technology in a manufacturing setting. The primary research goals were assessment and better understanding of a) the use of collaborative tools in a manufacturing (field) setting and $b$ ) the impact of this use on team performance and effectiveness, based on a comparison of pre- and post-intervention measures. An additional goal was to provide a collection of practical recommendations to potential manufacturing users and analysts, who may be considering using these tools in today's growing global economy.

\section{The research setting and approach}

In this research, the University of Michigan (UM) worked with an industrial partner, Auto $1^{\mathbb{W}}$, to deploy and assess the performance and impact of network-based tools for a geographically-distributed, engineering team, CAR. UM conducted interviews and administered a voluntary, web-based, pre-intervention survey to identify collaboration needs and to determine baseline, collaboration conditions. A suite of collaboration tools was recommended to Auto 1. The CAR team management agreed to the deployment and assessment of a tool for synchronous viewing of engineering drawings and documents, Microsoft (MS) NetMeeting 2 (NM). In conjunction with the Auto 1 Information Technology (IT) group, UM trained key CAR team personnel upon NetMeeting deployment. After 6 months of use, a second survey was administered. UM used the two surveys to analyze the adoption and impact of network-based, collaborative tools on the team's performance. Using this analysis, previous research, and follow-up questions to Auto 1 management, UM addressed the meaning of the results in terms of the adoption, use, and impact of collaboration tools, techniques for assessment and evaluation of collaboration tool use, and practical recommendations for users.

\section{Tool use and impact}

Although the analysis of the pre- and post-intervention surveys showed that a small proportion $(1 / 3, n=34)$ of the team used NetMeeting, NM users described a positive impact of this tool on their collaborative work. NetMeeting use reduced some of the difficulties indicated in the requirements analysis, such as interruptions in midconversation to send email attachments of drawings. With respect to the low adoption rate, results suggested a key feature of NetMeeting, specifically synchronous application sharing, was not as valuable as expected for collaborations that spanned time zones; but, it was more widely used for local collaboration. Another factor was that workarounds already in use within the team were sufficiently successful to inhibit exploration and

\footnotetext{
${ }^{1}$ Pseudonyms are used for the corporation, Auto 1, and its elements, e.g., CAR.

${ }^{2}$ Any commercial product identified in this document is for the purpose of describing a collaborative software environment. This identification does not imply any recommendation or endorsement by NIST.
} 
adoption of alternative collaboration tools, even when these tools had desirable features as identified in the collaboration requirements analysis.

\section{Assessment techniques, challenges and observations}

Over the course of one year at Auto 1, UM researchers used several techniques and approaches to assess and evaluate the use and impact of collaborative tools. They made numerous visits to CAR team sites, including European locations to conduct face-to-face interviews and training sessions. In collaboration with the Auto 1 IT group, UM conducted one-on-one training of NM and tracked deployment consequences for six months. Finally, UM developed, administered, and analyzed two surveys.

These efforts were conducted within a dynamic environment that threatened support for this research at Auto 1. There was continual uncertainty with respect to the ultimate success of the CAR team's product. There was also uncertainty regarding the legal relationship between Auto 1 and the University of Michigan concerning the overall research project with respect to intellectual property rights. Additionally, halfway through the research period, the Auto 1 vice president, who had invited and nurtured the research project, left the company. Even though the deployment and evaluation phases of the project were ultimately completed, these experiences reinforced the known difficulties in conducting this type of research in a business environment [6].

Observations from these efforts included:

- Conducting this type of research in a corporate environment, within actual engineering teams, required enormous cooperation and compromise to accommodate competing priorities.

- In the Auto 1 corporate environment, where IT resources were strained, the research team expended a great deal of effort attempting to bring a critical mass of users to a minimum level of competency with the target collaborative tools. With additional support from the Auto 1's IT group, more effort could have been expended directly on observation of the tools in use or enhancing practices with the tools.

- The use of surveys had the advantage of gathering data broadly and at relatively low cost. However, low response rates reduced the capacity to draw useful conclusions.

- Overcoming inertia in organizational settings is very difficult. For instance, if a solution to a collaboration issue already existed, there was little incentive for participants to change to another solution, even with anticipated improvements.

- A high-level champion in the field is vital for success of the implementation [6] and of the research. The "champion" at Auto 1 played an essential role in negotiation across the two divisions from which CAR team members originated. Momentum on this project slowed considerably when that high-level champion left Auto 1.

- UM's status as outsiders allowed them to probe the CAR team members to reveal practices and procedures that the engineers themselves, and Auto 1 personnel, did not have the time or inclination to explore. However, as outsiders, researchers confronted a very steep learning curve to understand the content of the engineering work. The effort expended on mastering the elementary aspects of the engineers' tasks could have been allocated to a more detailed examination of performance of the tasks and the relationship between these tasks and collaborative tools. In this way, inclusion of 
a veteran automotive engineer, specifically one from Auto 1, on the research team might have accelerated understanding of what the CAR team was trying to do. Yet, in the time- and resource-starved environment where CAR team members worked, it was unlikely that CAR team management or Auto 1 would have consented to an engineer/designer acting in a formal liaison role.

- It is important to anticipate competing demands in this type of research. Specifically, adoption of novel tools with accompanying novel practices is not something that unfolds on a time scale consistent with most projects. Therefore, researchers will confront the need to perform some level of "pump priming" - that is, there has to be some level of collaboration-tool adoption and use to produce behavior and outcomes that can be used to evaluate the impact of collaborative tools. Realistic determination of resources required to jump-start use, particularly where none currently exists, is difficult.

Practical recommendations for managers and engineers include:

- Selected collaborative tools must meet a specific need to merit the effort of deployment, adoption, and subsequent use. For example, the requirements gathering effort conducted early in this project, successfully highlighted difficulties experienced by the CAR team members when attempting to do cross-site work. At least for some of the CAR engineers, as indicated by adoption and use rates, the set of introduced collaborative tools met the identified needs. Yet, for many, the collaborative tool still imposed too great a burden to learn and master, relative to the perceived benefits.

- Successful deployment of collaborative tools requires a coordinated approach that involves the IT support organization at an early stage.

- Targeted changes in work practices, such as the adoption and use of collaborative tools, need adequate support to ensure success. In this study of NM adoption and impact, engineers were asked to master both the tool itself and also the choreography of working with a distant colleague via the tool. The significant investment of one-onone training surrounding the introduction of NetMeeting was successful for individual users. However, it was not conducted on a broad enough scale to create a critical mass of use for the team in the timeframe of this study.

\section{Concluding remarks}

The primary goals of this research were an assessment and better understanding of the adoption, use, impact, and evaluation of collaborative tools in a manufacturing setting. Because the collaborative tool intervention was specifically targeted to needs identified in the requirements gathering phase, a primary research expectation was to find a positive impact of tool use. While there was some indication of improved team performance with use of the tools, the overall adoption did not meet expectations. Although results were mixed, assessment of collaborative tool intervention is often difficult, even at the very coarse granularity of "success" and "failure" [1]. Therefore, one important outcome of this research is the well-documented case study [16], so that over time results can be interpreted more fully, and future research and collaborative tool deployments can draw from it. 


\subsection{Introduction}

This report summarizes a case study of a collaborative tool intervention within a geographically-distributed, product-development team of an automotive parts supplier. This report serves as a companion document and overview to the 170+ page report of that study, prepared as the final report to the National Institute of Standards and Technology (NIST) by the University of Michigan (UM), A Manufacturing Collaboratory Case Study [16]. Additionally, it is our intention that this document provide a portal to the abundant detail found in the final (full) report. Therefore, section references from the full report [16] are given throughout this document for the interested reader.

The primary research goals of the study were assessment and better understanding of a) the use of collaborative tools in a manufacturing (field) setting and b) the impact of this use on team performance and effectiveness, based on a comparison of pre- and postintervention measures. An additional goal was to provide a collection of practical recommendations to potential manufacturing users and analysts, who may be considering using these tools in today's growing global economy.

\subsection{Background}

Members of NIST's Manufacturing Engineering Laboratory and UM's Collaboratory for

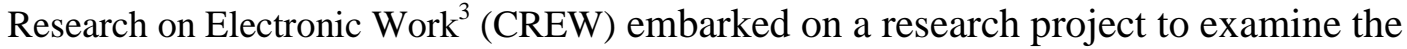
deployment of collaboration technology in a manufacturing setting. This research complements the work that NIST's Manufacturing Engineering Laboratory is doing in collaboration technologies and extends earlier work by UM in collaboratories. UM provided expertise in collaboratories, having deployed existing or prototype applications in real field settings, group behavior, and principles and methods of human-centered design, [16 (Sec. 1.2)]. NIST provided expertise in manufacturing, engineering, and collaboration tools for manufacturing.

Both organizations recognized the need for manufacturers to understand how to best use collaboration technologies. Field studies are an excellent way to generate this knowledge. However, reports on field studies typically provide anecdotal conclusions that can be difficult from which to learn. While we knew that conclusive results would be difficult to obtain when working in an area with numerous variables, we expected much useful information from a well-documented, case study. The UM final report, "A Manufacturing Collaboratory Case Study” [16], is that well-documented, case study.

\subsection{Research summary [16 (Sec. 2)]}

In this research, UM worked with an industrial partner, Auto $1^{1}$, to implement and assess the performance and impact of network-based tools for a geographically-distributed, engineering team, CAR. UM conducted interviews and administered a voluntary, web-

\footnotetext{
${ }^{3}$ Part of the School of Information, University of Michigan

${ }^{4}$ Pseudonyms are used for the corporation and its elements, e.g., Auto 1 and CAR.
} 


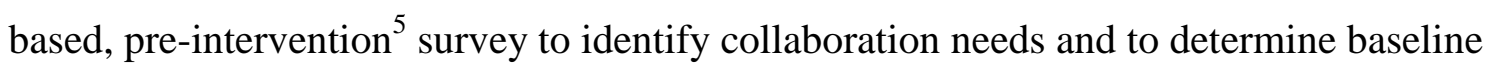
collaboration conditions. A suite of collaboration tools was recommended to Auto 1. The CAR team management agreed to the deployment and assessment of a tool for synchronous viewing of engineering drawings and documents, Microsoft (MS) NetMeeting (NM). UM also recommended deployment of a shared-calendar system and a presence-awareness system - neither of which were approved by CAR management for UM deployment, but had some spontaneous adoption that is discussed. UM trained key CAR team persopnel upon NetMeeting deployment. After 6 months of use, a second, post-intervention ${ }^{-}$survey was administered. UM used the two surveys to analyze the adoption and impact of network-based, collaborative tools on the team's performance. Using this analysis, follow-up questions to Auto 1 management, and previous research, UM addressed the meaning of the results in terms of the adoption, use, and impact of collaboration tools, techniques for assessment and evaluation of collaboration tool use, and practical recommendations for users.

To place this research in context, there are only two published studies on NetMeeting in the literature [11 \& 7]. In the case of Mark et al. [11], the study focused on room-toroom use of NetMeeting in an engineering-design setting within an aerospace manufacturing organization. Its use was primarily as an adjunct to audio conferences. Data were gathered on four teams over a period of three months. In the case of Finholt et al. [7], the study focused on targeted dyads of remote users performing software engineering within a telecommunications organization, again, covering a period of three months. The UM/NIST research effort with their industrial partner, Auto 1, extends this body of knowledge by focusing on a geographically-distributed, product-development team over a one-year period and generating a well-documented case study. [16 (Sec. 4.1)]

\subsection{Research environment}

The motivation for conducting this study was to understand the impact of network-based collaborative tools on the performance of a geographically-distributed work team. The significance of this research area is highlighted by the growing globalization of work, which requires an increase in coordinated activity across dispersed sites and employees, e.g., $[4,13]$. Specifically, in the manufacturing sector it is becoming common practice for engineers from different backgrounds and at different locations to combine their efforts to produce novel products. The UM/NIST strategy to assess the effects of modern communication technologies for collaboration was a detailed examination of a geographically-dispersed, engineering team.

With the cooperation of the industrial partner, Auto 1, UM identified a collaborative product-development group [16 (Sec. 2.1)], the CAR team. The team spanned four countries, three continents, and two divisions of the company. The CAR team had common characteristics of multi-site engineering projects:

\footnotetext{
${ }^{5}$ The "pre-intervention" identifier is synonymous with "Time 1." These identifiers refer to the period of time prior to the deployment of NM.

${ }^{6}$ The "post-intervention" identifier is synonymous with "Time 2." These identifiers refer to the assessment period approximately six months after the initial deployment of NM.
} 
- The engineers were located all over the world (Europe, the United States, and Australia) and regularly confronted barriers of distance and time.

- The members of the team had to cope with cultural, linguistic, procedural, and technological differences - often with little allowance or guidance from the organization.

- The infrastructure to support the work of the team was dynamic, including the underlying network technologies and data formats that required ongoing adjustment and accommodation by the engineers.

- The team brought together engineers with diverse technical backgrounds. This required that problems and solutions be expressed via mutually understandable terms and concepts.

- The team was under extreme pressure to meet scheduled deliverables. This pressure was compounded by fact that key milestones and product characteristics were under constant negotiation with prospective customers.

- The success of the team had strategic importance for the company, since the potential market for the product in development was estimated at over \$1 billion.

- The team was newly assembled to produce the target automotive subsystem and did not have a prior history of working together.

- The team was the initial effort by the larger organization to join expertise across corporate divisions to develop an innovative product.

Against this backdrop, UM identified and proposed a collaboration technology deployment strategy based on rigorous analysis of the team's requirements. Then UM instrumented the deployment to assess the impact of collaboration technology use on the team's effectiveness. [16 (Sec. 2.1, 2.3)]

\subsection{User-centered Design: Surveys and Interviews (Time 1)}

To understand the collaboration needs of the CAR team and to establish a baseline of collaboration conditions, UM developed and administered a web-based survey to each of the 50 CAR team members and interviewed a subset (24). The pre-intervention survey and interviews were designed to capture an initial data set of team conditions against which future data (from the post-intervention survey) could be compared.

\subsection{Interviews [16 (Sec. 2.2.2)]}

The selected subjects for the interviews were individuals whose point of view or insight might be particularly helpful because of their position or skill set. The interviews with the team members and management addressed:

- Work roles and background

- Identification of local and remote collaborators

- Current means of communicating with remote collaborators

- Opportunities for remote collaboration

Following user-centered, design principles, e.g., [5], UM personnel addressed these topics to gain an understanding of the CAR team members' current work practices. 
Questions were aimed also at uncovering established norms and potential barriers to future collaborative tool introduction. The interviews were semi-structured in format. This means the interviewers allowed for conversation with the informants, so that informants could expand on topics most relevant to themselves and open the possibility for gaining additional, unforeseen information.

\subsection{Pre-intervention Survey [16 (Sec. 2.5)]}

All 50 members of the team were invited by their management via email to participate in the study and complete the web survey. No incentives for participating in the study were offered. Thirty-four out of $50(68 \%)$ employees completed the pre-intervention survey from 6 geographically-distributed sites, in 3 countries. Most respondents (65\%) came from European sites (18 from Germany, 4 from France, 12 from the United States) [16, Appendix F].

The web survey consisted of 48 questions covering:

- Background items [16 (Sec. 2.5.1)]

- Demographic information

- Job satisfaction

- English speaking skills

- Location

- Workstation

- Independent variables [16 (Sec. 2.5.2)]

- Technology adoption motivation

- Work-related communication patterns

- Non-work related communication patterns

- Workflow

- Outcome measures [16 (Sec. 2.5.3)]

- Coordination and performance

- Organizational citizenship

- Trust

- Group identification

\subsection{Collaboration Requirements [16 (Sec. 2.4, 3.1)]}

This section describes the themes, related work-practices, and barriers that were reported by the CAR product-development team during the requirements gathering phase of the study.

These themes were derived by qualitative analysis of the interviews to determine the most frequently mentioned issues in distributed work within the CAR team. This was accomplished using standard practices for qualitative data analysis $[8,9,12]$. This involved constructing inductive code categories first by reading through the background interviews and creating an extensive list of all the reported issues, then clustering these issues into categories of related statements. Descriptive statistics from the pre- 
intervention survey supported three themes: reliance on email, coordination issues, and maximizing synchronous time.

Heavy reliance on email. The following quote from one respondent was representative of attitudes towards email as a collaboration tool: "The most valuable tool is email... because it's something that's always there."

To overcome the coordination issues inherent in remote collaboration, CAR team members had established norms to help them expedite their work. While these norms were resourceful adjustments to dispersed teamwork, they were often cumbersome solutions for collaborative work problems. Team members described using email foremost as a tool for contacting remote team members, as well as, the primary means for exchanging task requests, data, reports, designs, and sketches. As needed, these items were exchanged as attached files.

Yet, these practices fell short of respondents' needs. Traveling team members experienced difficulties accessing their email accounts remotely. File transfers were sometimes slow and caused delays in work. File transfers were often slowed due to large file sizes, generally Computer-Aided Design (CAD) drawings. Additionally, encryption standard incompatibilities led to cumbersome security procedures. Finally, email did not offer a rich medium for information exchange. For instance, email did not easily allow users to identify or describe visual details, which are common in engineering design work.

Coordination issues. Difficulties in remote collaboration are often due to inherent coordination problems of geographically-distributed team members [14]. For example, typical team coordination activities, such as scheduling meetings, are often slowed by large time zone differences.

In this study, respondents expressed frustration due to: 1) difficulty in coordinating with team members located in other time zones, 2) limited synchronous time they shared with co-workers located in other time zones, and 3) the resulting work delays. On average, team members reported 4 delays each month, with $90 \%$ encountering delays of a day or more when working with remote collaborators. Furthermore, team members described difficulty in scheduling common meeting times with both local and remote co-workers. Often, problems in scheduling stemmed from difficulties locating local co-workers who traveled frequently. Yet, problems in locating and scheduling were more pronounced with remote rather than local co-workers. Coordination issues were correlated statistically with both delays in work and difficulties establishing trust with remote team members.

Maximizing the effectiveness of synchronous time. One of the challenges facing this geographically-distributed team was the scarcity of common work times. CAR team members indicated experiencing difficulty scheduling common work time with remote team members due to limited overlapping work hours. 
This theme is somewhat interwoven with the 'coordination' and 'reliance on email' themes, in that, often team members coordinated synchronous meetings via email. For example, one participant mentioned that one "may say (over email) 'call me this afternoon, I have to talk to you about' such and such."

Participant team members most commonly used the telephone for synchronous work, while a few team members used shared application tools, and fewer still used video conferencing. Typically, team members conducted meetings via the telephone in order to: discuss coordination issues, conduct design review, acquire further information or details, and clarify issues mentioned in email. Team members explained that they would like to use visual aids to enhance such meetings. Some team members sent faxes to exchange sketches or other materials simply and quickly. In addition, team members who discussed sketches, designs, or visual materials, expressed the desire to have tools to improve mutual understanding of the material, such as being able to point to areas in a drawing under discussion.

Additionally, team members traveled between North America and Europe in order to facilitate working together. Language differences caused some communication problems that were best handled with face-to-face meetings. Email and telephone interactions were described as working better after there had been an initial, face-to-face meeting. Finally, Americans often used overtime to synchronize work with European counterparts. However, this practice had limited utility as the labor laws of one of the European countries involved in the study constrained their citizens to an 8-hour workday.

\subsection{Collaborative tools recommendation, deployment, and use [16 (Sec. 3.2)]}

The requirements analysis resulted in the recommendation of three technology interventions: a tool for synchronous viewing of engineering drawings and documents (e.g., Microsoft NetMeeting); a tool for sharing calendar information (e.g. Microsoft Outlook); and a tool providing presence-awareness information (e.g., ICQ ${ }^{2}$ ). Only one direct intervention was pursued due to resource constraints within Auto 1 and legal concerns about the export of personal data - such as schedule information - outside the European Union. That intervention was a supported introduction of NetMeeting.

In conjunction with the CAR team management, UM researchers identified a subgroup of engineers for early adoption of NetMeeting and arranged a schedule of collaborative tool implementation. Members of the subgroup were selected because their management believed they had an immediate need to collaborate with remote colleagues. Fifteen team members in two U.S. and two European locations were trained, involving a 15 minute introduction followed by a 30 minute guided use of NetMeeting in a domain-relevant, document-sharing session. Simultaneous training sessions across sites were conducted so that the team members could engage in remote collaboration in the course of their training. As a follow-up to the NetMeeting training, UM researchers sent unsolicited weekly hints to subgroup members on the use of NetMeeting and requested feedback via email on the CAR team's weekly use of the collaborative tools.

\footnotetext{
${ }^{7}$ http://www.icq.com/
} 
Although the CAR management team elected not to adopt a presence-awareness or calendar tool as part of this research effort, there was some ad hoc adoption among CAR team members. The adoption of these tools is addressed in [16 (Sec. 4.1.2)].

\subsection{Post-intervention survey and follow-up interviews [16 (Sec. 2.6)]}

The post-intervention survey consisted of 42 questions in 5 sections: technology adoption, communication patterns, coordination, performance and trust. 34 participants (68\% of the team) responded to the post-intervention survey. Approximately a third of these respondents had also completed the pre-intervention survey. Of these, $70 \%$ were located in Europe and 30\% were located in offices in the United States.

While the basic format and sections of the second survey were the same as the first survey, several changes were made. These changes were made to avoid redundancy and to reduce the amount of time required to complete the second survey. Feedback about the first survey indicated that many respondents required more than the promised 30 minutes to complete the survey. Additionally, a significant number of respondents skipped questions at the end of the survey.

\subsection{Items eliminated [16 (Sec. 2.6.1)]}

Questions regarding the participants' background and workstation, non-work relationships and friendships, and group identification were eliminated. Background and workstation information had remained essentially unchanged over the course of the study. Questions regarding team members' non-work communication patterns were eliminated due to the excessive time required to complete these questions and to the low response rate from the first survey. The group identification questions were removed due to low response and the fact that responses to these questions did not vary. That is, these questions were uniformly answered in positive terms, leading analysts to question the validity of these items for measuring group members' actual sentiments.

\subsection{Items altered [16 (Sec. 2.6.2)]}

The questions about work communication patterns were changed so that they would no longer require participants to repeatedly answer the same questions regarding up to 15 of their co-workers, as in the first survey. Instead, respondents were to indicate the number of co-workers with whom they communicated on a daily basis.

\subsection{New items [16 (Sec. 2.6.3)]}

The first survey asked individuals to discuss their motivation to adopt technology. In contrast, the second survey asked about actual tool use. In the second survey, questions were added to assess the impact of the collaboration tools that had been implemented during the study. Specifically, new questions included:

- "Did the use of any of these collaborative tools change the manufacturing design process (i.e., the way you and your team went about working on the CAR 
project)?" If participants answered "Yes", they were asked to respond to the following questions:

- "The use of these collaborative tools improved the quality of the product"

- "The use of these collaborative tools improved the efficiency of the design process"

- "The use of these collaborative tools improved the speed of the design process"

\subsection{Results}

To better understand the changes that occurred in the CAR team during the research period, UM analyzed the two surveys in three ways. First, the individual items in the surveys were analyzed and compared. This gave a flavor for changes occurring between the pre- and post-intervention surveys. The findings are given in section 7.1. Second, the difference in mean scores on outcome variables was analyzed using a paired-sample t-test [3]. This test compared identical measures in both surveys. The findings are presented in section 7.2. Third, correlation analysis was used to determine whether variables were associated with one another. These findings are discussed in section 7.3.

\subsection{Time 1 and Time 2 Survey Findings [16 (Sec. 3.3)]}

\section{Communication Patterns-Time 1 [16 (Sec. 3.3.3 \& Figures 1.1, 1.2)]}

With respect to communication, team members were asked about their work contact patterns, frequency of communication with other team members across various media, and travel to other sites.

- On average, participants reported communicating often with 5.8 (s.d. = 5.39) coworkers.

- The most frequently used modes of communication were face-to-face (83\% reported high use), email (35\% reported high use), and telephone (29\% reported high use).

- European team members reported higher levels of telephone and email than their U.S. counterparts.

- Across all modes of communication, respondents contacted the German product development site most often. This was the site where the design work was occurring.

Communication Patterns-Time 2 [16 (Sec. 3.3.4 \& Figures 2.1, 2.2)]

- Patterns of media use were similar to those found at Time 1, except that the percentage of participants reporting high frequency of face-to-face communication was lower at Time 2.

- Differences in media use between U.S. and European Union (EU) persisted: EU used the telephone and collaboration tools more often, whereas U.S. used more face-to-face communication.

- Collaboration-tool use had spread to more than half the team. 
- Like Time 1, respondents contacted the German product-development site most often. However, unlike Time 1, respondents traveled to the German manufacturing site most often. This change from the first survey likely reflects the downstream production process occurring at Time 2.

- Respondents reported traveling half as often as in Time 1.

In terms of collaboration-tool use, American respondents did not use collaboration tools at all, compared with $42 \%$ of European respondents. Half of the European respondents reported using collaboration tools regularly, between 1 and 4 times per week. 8\% used these tools very frequently. It is possible that the collaboration-tool use in Europe reduced the need for face-to-face communication for European respondents, as compared to Americans. In fact, a post-training interview suggested that European team members often used NetMeeting to hold meetings between office buildings at the German productdevelopment site.

\section{$\underline{\text { Technology Adoption-Time } 1 \text { [16 (Sec. 3.3.5 \& Figure 1.5)] }}$}

The overall level of interest in using new technology for collaboration was high:

- $100 \%$ of U.S. respondents reported a strong desire to adopt a shared, mark-up tool, compared with $55 \%$ of European respondents.

- $83 \%$ of U.S. respondents reported a strong desire to adopt availability and presence tools, compared with $59 \%$ of European respondents.

- $83 \%$ of U.S. respondents reported a strong desire to adopt an electronic-calendar tool, compared with $55 \%$ of European respondents.

\section{Technology Adoption-Time 2 [16 (Sec. 3.3.6 \& Figure 2.6)]}

Whereas the technology adoption items in the Time 1 survey assessed the participants' inclination to adopt new tools, the items at Time 2 assessed their actual use of these tools, and the impact it had on their work.

- High calendar use, low application-sharing and presence-awareness tool use.

- $50 \%(n=32)$ of all respondents agreed that NetMeeting had an impact on their work, with most agreeing that it improved the efficiency and speed of the design process.

The overall use of new technology for collaboration was high for some tools and not others. For instance, at the time of the second survey, all $(100 \%, \mathrm{n}=34)$ participants were using the electronic calendar. However, only $38 \%$ used a presence-awareness tool or an application-sharing tool. Sixty-two percent of respondents indicated that they did not use NetMeeting at all in the last 6 months. Regular use of the shared-application tool was positively correlated with agreement that collaborative tools had changed the design 
process. Adoption of the calendar and presence-awareness tools was not significantly correlated with this variable.

To further interpret the effect of collaboration-tool use, participants were asked whether the use of these tools changed the manufacturing design process, and how the tools had an impact.

- $50 \%$ of respondents $(n=32)$ reported that the collaboration tools they used had changed the design process.

- $59 \%$ agreed that the speed of the design process had improved.

- $82 \%$ agreed that the efficiency of the design process had improved.

- $47 \%$ agreed that that the quality of the design process had improved.

Thus, of those who perceived a change in the design process, a majority agreed that it was a positive change. Additionally, the tools mostly affected the efficiency and speed of the design process, with a lesser effect on the quality of the work produced.

\section{$\underline{\text { Workflow-Time } 1[16 \text { (Sec. 3.3.7 \& Figure 1.6)] }}$}

CAR team members indicated the percentage of their work that fit into each of the following types of workflow:

- Independent: co-workers perform work separately.

- Sequential: work flows between co-workers in one direction.

- Reciprocal: work flows between co-workers in a "back and forth" manner over time.

- Team: co-workers diagnose, problem-solve and collaborate as a group at the same time.

On average, the greatest mean percentage of the respondents' work was conducted independently $(n=34$, mean $=30 \%$, s.d. $=22.49)$.

\section{Workflow-Time $2[16$ (Sec. 3.3.8 \& Figure 2.8)]}

- On average, the greatest mean percentage $(29 \%$, s.d. $=23.42)$ of the respondents' work was conducted as team work, in contrast to Time 1, where independent work had the highest mean percentage.

- In general, team members seemed to be spending more time in reciprocal and teamwork than independent at the conclusion of the study. The mean percentage of sequential work did not change.

\section{Working Relationships-Time 1 [16 (Sec. 3.3.9 \& Figures 1.7, 1.8, 1.9)]}

The surveys addressed two types of issues regarding relationships at work: group identification and trust. 
- In general, group identification was high. For example, a larger percentage of respondents (96\%) believed that criticism of the project was taken as an insult by local, rather than by remote team members (37\%).

- Overall levels of trust in both local and remote team members were high. However, respondents reported consistently lower levels of trust for remote team members than for local team members.

Working Relationships-Time 2 [16 (Sec. 3.3.10 \& Figures 2.9, 2.10)]

- Group identification was not measured at Time 2 [16 (Sec. 2.6.1].

- Overall levels of trust seemed to have remained at the same high level from the beginning of the study.

- Although not statistically significant, the authors of the full report reported an increased disparity at Time 2 between trust in local and remote co-workers.

- Trust was lower for remote team members than for local team members.

Coordination, performance and organizational citizenship-Time $1[16$ (Sec. 3.3.11 \& Figures 1.10, 1.11, 1.13)]

- Respondents reported greater difficulty in coordinating their work with remote rather than local co-workers.

- There was a positive correlation between receiving timely information about changes in current plans from remote co-workers and reported trust in remote coworkers.

- Team members indicated experiencing delays in work involving both local and remote co-workers.

- A greater number of respondents (50\%) reported a high frequency of delays (four or more delays in the previous month) involving local co-workers than with remote co-workers $(42 \%)$.

- Most respondents $(90 \%)$ reported a high average length of delay (one to several days delay) in work involving remote co-workers. In contrast, less than half of the respondents (48\%) reported such lengthy delays with local co-workers.

- Difficulty scheduling common meeting times with remote co-workers was positively correlated with frequency and average length delays involving remote co-workers.

- In general, respondents reported high levels of organizational citizenship.

Coordination, performance and organizational citizenship-Time $2[16$ (Sec. 3.3.12 \& Figures 2.11, 2.12)]

- As at Time 1, participants continued to have greater scheduling difficulties with remote rather than local co-workers.

\footnotetext{
${ }^{8}$ Citizenship behaviors are those behaviors relating to cooperation with co-workers, helping team members, creating a favorable environment, and so on $[2,10,15]$.
} 
- A high frequency of delays was more common with local co-workers, as at Time 1 .

- The number of high frequency delays decreased at Time 2.

- High length of delay was more common with remote than local co-workers, as at Time 1.

- Mean length of delay decreased.

- Time 2 organizational citizenship levels were slightly higher than at Time 1.

\subsection{Comparing Means at Time 1 and Time 2 [16 (Sec. 3.4 \& Appendix I - Tables 8, 9)]}

Using a paired-sample t-test to compare before and after measures on the same sample yielded only one significant difference. The mean percentage of time spent in independent work changed significantly $(\mathrm{p}<.01)$, from $32.5 \%$ at Time 1 (standard deviation (s.d.) $=22.49)$ to $21.25 \%$ (s.d. $=20.71)$ at Time 2 . Some other variables displayed relatively large differences in means, however, these differences were not statistically significant.

One reason these findings may not be completely accurate in describing changes from Time 1 to Time 2 is the nature of the statistical test. The paired-sample t-test eliminates data from participants who did not respond in both Time 1 and Time 2 for each variable in question. Because not all survey participants completed both surveys or answered all the questions of both surveys, the sample size for the paired-sample t-test was often reduced to $1 / 3$ of the total participants who completed the surveys. The smaller the sample size, the greater an effect must be in order to be determined as statistically significant. Thus, differences in means that may seem large (like 17.92 total number of days spent visiting other sites at Time 1 compared with 12.25 at Time 2), are not statistically significant. Furthermore, the means used for the paired-sample t-test represent only that data collected from the subgroup of participants who completed the item both times. Thus, the entire Time 1 survey mean for total number of days spent visiting other sites was actually 17.71 at Time 1 (approximately the same as the mean of 17.92 used for the statistical test) and 8.19 at Time 2 (much lower than the subgroup's mean of 12.25).

\subsection{Relationships among variables [16 (Sec. 3.5 \& Appendices G, H)]}

UM used correlation analysis to determine whether variables were associated with one another. All the correlation results listed in the following section are significant at $\mathrm{p}<$ .05 unless stated otherwise.

\section{Impact of collaboration tools [16 (Sec. 3.5.1)]}

- Quality and efficiency improvements due to the collaborative tool intervention were positively correlated with one another, as were efficiency and speed improvements. Thus, the impact of NetMeeting may be described as a general 
improvement. And, that impact can be characterized more specifically with respect to coordination, performance, and trust among co-workers (see below).

- Application-sharing tool adoption was positively correlated with agreement that NetMeeting had changed the design process.

- There was a positive correlation between the number of local and remote coworkers that participants communicated with daily, and perceiving a change in the design work as a result of the implementation of NetMeeting. Thus, the greater the number of team members with whom one communicated with on a daily basis, the more likely one is to have perceived an impact on design work as a result of tool implementation.

Coordination $[16$ (Sec. 3.5.2)]

- Difficulties with one type of coordination were associated with difficulties in other coordination activities. Local and remote scheduling difficulties were positively correlated. Receiving timely information about changes in plans from local and remote co-workers was correlated positively. And, difficulty finding local and remote co-workers was correlated positively. Furthermore, individuals who perceived scheduling difficulties with local co-workers also perceived greater difficulty in receiving information from remote co-workers.

- There was a positive correlation between the number of local co-workers with whom one communicated and receiving timely information from local coworkers. It is possible that one's position in the organizational hierarchy may confound this association. For instance, senior team members, particularly managers, are often likely to communicate with a larger number of employees than do junior engineers. Additionally, they typically command faster response rates.

- As a result of the NetMeeting intervention, perceptions of efficiency and speed improvements in the design process, were positively correlated with the perception that remote co-workers provided timely information regarding changes in plans. This finding supports the notion that NetMeeting provided value in working with remote co-workers.

- Coordination difficulties were associated with decreased individual citizenship behavior and perceptions of other's citizenship behavior. For instance, difficulty in scheduling meetings with local co-workers was positively correlated with difficulty in passing information to remote co-workers. It was also positively correlated with difficulty in receiving information from local and remote coworkers. Therefore, as individuals perceived fewer barriers to coordinating with local co-workers, they tended to perceive greater organizational citizenship from their team members.

\section{Performance [16 (Sec. 3.5.3)]}

- Length and frequency of delay were significantly correlated to one another. For instance, certain team members may be more likely to experience frequent, long 
delays with both local and remote co-workers, while others experience few delays, which are of shorter duration.

- There was a positive correlation between frequency of local, face-to-face workrelated communication and average length of delay due to remote co-workers. This means that a greater incidence of face-to-face work with local co-workers was associated with shorter delays in work caused by remote co-workers.

- Coordination difficulties related to performance issues. For instance:

- The greater ease one had in locating co-workers, the shorter the perceived, average length of delay.

- Increased local coordination was associated with shorter delays in obtaining work input from local team members.

- The more participants rated local co-workers as giving them timely information, the shorter the average delays they experienced as a result of local co-workers. And, similarly, for remote workers.

Therefore, it is possible that alleviating coordination problems may contribute to performance improvements. The next two items examine the specific relationships between collaboration-tool use and coordination, and collaboration-tool use and performance.

- Coordination and NetMeeting use. There was only partial support for a relationship between collaboration-tool use and improvements in coordination. Regular use of NetMeeting was not significantly correlated with coordination. However, of the team members who perceived that the design process had been changed by NetMeeting use, coordination improvement was associated with perceptions of efficiency and speed improvements in the design process as a result of NetMeeting. These findings suggest that the relationship between NetMeeting use and coordination improvements is unclear, and deserves further investigation.

- Performance and NetMeeting use. There were mixed results supporting a relationship between the perceived impact of NetMeeting on the design process and performance. Perceptions of improved efficiency due to the NetMeeting were associated with a lower number of delays caused by remote co-workers. Yet, indications that NetMeeting had affected the design process was correlated with a higher frequency of delays due to remote co-workers. Furthermore, agreement that NetMeeting improved the quality of the design process was associated with increased length of delay due to local co-workers. These findings indicate that the relationship between collaboration tool use and performance is not clear, because the indicators of that relationship are mixed, both positive and negative.

\section{$\underline{\text { Trust }[16(\text { Sec. 3.5.4)] }}$}

- Higher levels of trust in local co-workers were associated with higher levels of trust in remote co-workers.

- There was no specific relationship between NetMeeting use and trust. However, perceptions that the use of NetMeeting improved the speed of the design process 
were positively correlated with increased trust in remote co-workers and increased trust overall.

- Ease of coordination was related to increase in trust. For instance, greater ease in locating distant co-workers was associated with greater trust in local and remote team members. Also, receiving timely information about changes in plans from local and remote co-workers was positively correlated with increased trust in local and remote co-workers.

- There was a positive relationship between performance and trust. For example, lower average length of delays was associated with increased levels of trust in local co-workers.

- Increased organizational citizenship related to an increase in trust. For instance, receiving useful information from local and remote co-workers was positively correlated with local, remote and overall trust.

These findings support the study's original assumptions about the importance of being able to coordinate with and rely on team members to build and maintain trust in the team. Thus, understanding how collaboration tools play a role in affecting the coordinationperformance-trust triangle remains a question worthy of investigation.

It is more difficult to assess the exact relationship of collaboration-tool use and trust. While there was no specific relationship between NetMeeting use and trust, the belief that collaboration tools improved the speed of common work was related to increased trust.

\subsection{Discussion [16 (Sec. 4)]}

Through a variety of mechanisms, the CAR team did experience a significant increase in use of collaborative tools over the period of the study. Although adoption of NetMeeting was lower than anticipated, it did occur. CAR managers launched an effort independent of the research study to encourage shared-calendar use. Additionally, there was some spontaneous adoption of presence-awareness tools. The analysis of this use led to a number of critical insights. They can be categorized in terms of three contributions to understanding:

- The general role of collaborative tools in geographically-distributed teams and the specific impact on geographically-distributed manufacturing engineering teams

- Techniques and methods for assessment and evaluation of technology-related changes, particularly related to the introduction and adoption of collaborative tools

- Practical recommendations to organizational leaders in the manufacturing sector, and more broadly, about how and when to introduce collaborative tools to ensure maximum success

\subsection{Adoption and use of collaborative tools [16 (Sec. 4.1.1)]}

To summarize, fifteen members of the CAR team, who were judged by their management to have the greatest need for NetMeeting features, were trained on NM use. This strategy succeeded in exposing NetMeeting to a significant fraction of the CAR engineers. For example, from a baseline level of no use, $36 \%$ of the CAR team reported some level of NetMeeting use by the end of the study period. However, all reported users were from 
European sites. [Note: Researchers were aware of some regular use at U.S. sites, but these engineers did not respond to the post-intervention survey.] A small number of team members, 6\%, reported regular use of NetMeeting. No formal deployment effort was involved for the other two recommended collaborative tools, shared-calendar and presence-awareness applications. By the end of the study, 97\% of the CAR team reported some level of shared-calendar use. And, a large number of team members, $82 \%$, reported regular, shared-calendar use. Adoption of presence-awareness tools was much lower, with some reported use by $36 \%$ of respondents by the end of the study.

An unresolved question is why adoption of the calendar tool was so much broader than the adoption of NetMeeting and presence-awareness tools. Prompted by the requirements analysis, the lead managers within the CAR team decided to recommend use of a sharedcalendar tool. However, NetMeeting was also endorsed strongly by management. One factor in the differential adoption rates may have been the types of capabilities provided by the two tools. For the CAR team, there was a smaller number of engineers who benefited from the relatively specialized capabilities of NetMeeting versus those who benefited from the relatively generic capabilities of a shared-calendar tool. That is, scheduling is a more universal need. By contrast, a tool for application sharing appeals mainly to those workers who must collaborate with distant colleagues and must do so in real-time, as when viewing a common drawing or document. Finally, the manager of the one division observed that most of their work with EU colleagues was concentrated on the French manufacturing site - and not the German product development site. At the time of the study, the French site had very poor network connectivity. As a result, low NetMeeting use might not have reflected the team members' desire to use the tool, but rather reflected the low performance of the network links between the U.S. and the French location.

\subsection{Impact of collaborative tools [16 (Sec. 4.1.2)]}

When team members were asked about the collective impact of collaboration-tool use on changes in the manufacturing design process, the overall response was that $50 \%$ felt that changes had occurred. However, focusing on those team members who used the various tools, the numbers were different. For example, among NetMeeting users $66 \%$ reported change compared to $37 \%$ among non-users - while only $46 \%$ of calendar and $45 \%$ of presence-awareness users reported change. We conclude that NetMeeting use produced a more profound perception of change among users than either shared-calendar or presence-awareness use.

Of those team members who reported changes, $56 \%$ of NetMeeting users felt that efficiency and speed had improved, although only $22 \%$ felt that quality had improved. Among the shared-calendar users who reported changes, $83 \%$ felt that efficiency had improved, $75 \%$ felt that speed had improved, and 50\% felt that quality had improved. Finally, among the presence-awareness users who reported changes, $80 \%$ felt that efficiency had improved, $60 \%$ felt that speed had improved, and $60 \%$ felt that quality had improved. Therefore, it appears that the primary impact across all collaboration-tool use was on efficiency and speed, with a smaller impact on quality. 
Perceptions of efficiency improvements and perceptions of speed improvements were positively correlated with the perception that distant co-workers provided timely information $(r=0.72$ and $r=0.66$, respectively; both $p$ values $<0.05)$. This relationship suggests that use of collaborative tools did reduce some of the difficulties indicated in the initial round of interviews, such as interruptions in mid-conversation to send email attachments of drawings.

The analysis of critical performance outcomes, such as the frequency and duration of delays with respect to the collaboration tool indicated no specific relationships. Further, team members were asked about critical process issues, like frequency of travel. While frequency of travel did decline according to responses at the second survey administration, there was no significant association of this decline with use of the introduced collaboration tools. A division manager, when questioned about the reduced travel, attributed the reduction to the natural ebb and flow of travel corresponding with different periods of the manufacturing design cycle. That is, in his opinion, higher travel at the time of the first survey administration probably reflected a more intensive overall level of interaction between the European and U.S. sites than at the time of the second survey.

Finally, team members responded to questions about critical social and psychological factors, such as trust. Research expectations were that trust might be improved as distributed team members increased opportunities to establish common ground and overcome spatial and cultural barriers by using collaborative tools. However, there was no specific relationship between the use of collaborative tools and trust. There was a factor associated with collaboration-tool use, perception of improved speed, that was positively related to trust in remote co-workers, and overall increased trust, but not in local co-workers alone. This suggests that greater responsiveness plays a role in assessments of trustworthiness, and to the extent that collaborative tools improve responsiveness, they may be expected to influence perceptions of trustworthiness.

\subsection{Assessment techniques, challenges and observations [16 (Sec. 4.2)]}

The main focus of this research was characterization of the use and impact of collaborative tools within a distributed, engineering team. However, an important, secondary goal was the demonstration of techniques for assessment and evaluation of collaboration-tool interventions that could be generalized to other field settings.

Over the course of one year at Auto 1 UM researchers performed the following tasks:

- Made dozens of visits to CAR team sites, including two trips to European locations

- Organized three "all hands" meetings involving Auto 1 upper management, CAR team management, the Auto 1 IT organizations, and UM and NIST researchers

- Developed, administered, and analyzed two surveys

- Developed and implemented a complete technology deployment, including one-onone training, and tracked the consequences of the deployment for six months

These efforts were conducted against a corporate backdrop at Auto 1 that included:

- Uncertainty with respect to the ultimate success of the CAR team's product 
- Uncertainty concerning the legal relationship between Auto 1 and the University of Michigan concerning the overall research project, particularly with respect to intellectual property

- The departure, halfway through the research period, of the Auto 1 vice-president who invited and nurtured the research project

These difficulties in conducting the research reflect the first crucial lesson in terms of the assessment and evaluation strategy. Doing research in the field, within actual working teams, requires enormous cooperation and compromise. For example, UM wanted to introduce three collaborative tools. Because of Auto 1 legal and operational concerns, they were only allowed to introduce one tool.

Second, having negotiated access to Auto 1 on the basis of minimizing demands placed on the Auto 1 IT organizations, UM researchers were placed in the position of conducting the bulk of training and also debugging problems with applications, such as NetMeeting server failures. This meant that tremendous effort was expended to bring users to a minimal level of competency with the target collaborative tools and to address tool failures. Under other circumstances, that effort might have gone directly into observation of the tools in use or to enhancing practices with the tools.

Third, the surveys had the advantage of gathering data broadly at relatively low cost. However, low response rates and the small number of respondents for both surveys, reduced the capacity to draw useful conclusions. For example, the post-intervention survey showed no US-based NetMeeting users. However, interview and site visit experience suggested that there were regular NetMeeting users at U.S. locations.

Fourth, overcoming inertia in organizational settings is very difficult. For example, if a solution to a collaboration issue already existed, there was little incentive for participants to change to another solution. This was true for NetMeeting use. Frequent users of email attachments found NM to be a benefit to them, but those who used attachments infrequently did not use NetMeeting. Similarly, another subgroup used an applicationsharing tool other than NetMeeting. Since this solution was adequate for them, they did not find it necessary to change applications - this was communicated in a follow-up interview. This was true even though the lead engineer of this subgroup was perceived by management to be technology-savvy and an early adopter of technology. Learning the new tool was not a problem, but there was little incentive for him to change.

Fifth, UM's entrée to Auto 1 and early development of the project was greatly facilitated by the enthusiastic support of the Vice-President (VP) for Human Resources in one of the divisions. This VP played an essential role in helping negotiate across the two divisions involved with the CAR project. The significance of this influence reinforces the importance of a high-level champion within the field organization [2]. Momentum on this project slowed considerably when this high-level champion left Auto 1.

Finally, UM's status as outsiders allowed them to probe the CAR team members to reveal practices and procedures that Auto 1 personnel did not have the time or inclination to 
explore. However, as outsiders, they confronted a very steep learning curve to understand the content of the engineering work. Again, effort expended on mastering the elementary aspects of the engineers' tasks could have been allocated to more detailed examination of performance of the tasks - and the relationship between these tasks and collaborative tools. In this way, inclusion of a veteran automotive engineer, especially one from Auto 1, on the research team might have accelerated basic understanding of CAR team tasks. Yet, in the time and resource starved environment where CAR team members worked, it was unlikely that CAR team management or Auto 1 would have consented to an engineer/designer acting in a formal, liaison role.

\subsection{Practical recommendations [16 (Sec. 4.3)]}

While the CAR team's experiences could be considered representative of the universal difficulties and challenges confronted by multi-site engineering organizations, it was a single team within one organization. Therefore, any generalizations should be made with appropriate caution. Specifically, unique features of this research setting included the history of very autonomous divisions within Auto 1. This autonomy may have contributed to greater than normal difficulties in cross-division communication and cooperation. Also, the strategic importance of the CAR project, and the corresponding high visibility of the project within Auto 1, may have increased the team's motivation to perform. It is conceivable that the team performed to the point where the addition of collaborative tools had less impact than would be the case for a team not in the spot light. Finally, because of the cross-cultural interaction within the CAR team, multi-site collaboration issues may have been confounded with cultural and language issues. Although, most team members spoke and understood English very well.

With these caveats in mind, there are a number of practical recommendations that can be made based on the CAR team's experiences. These recommendations fall into two categories: a) advice to managers and engineers about adoption and use of collaborative tools; and b) advice for future researchers studying the impact of collaborative tools in engineering settings.

\subsubsection{Advice to managers and engineers [16 (Sec. 4.3.2)]}

The foremost conclusion of this study is that collaborative tools must meet a specific need to merit the effort of deployment, adoption, and subsequent use. For example, the requirements gathering effort conducted early in this project successfully highlighted difficulties, or constraints, experienced by the CAR team members while attempting to conduct cross-site work. At least for some of the CAR engineers, the set of introduced collaborative tools met identified needs. Yet, for many, the available tools still imposed too great a burden to learn and master, relative to the perceived benefits. That is, for those who interacted heavily with colleagues at distant sites, the collaborative tools that were introduced were well received. For others, existing solutions were sufficiently satisfactory to discourage investigation of alternatives or the infrequent interaction with distant colleagues did not prompt use of the new tools.

There were also a number of engineers who wanted to use the collaborative tools, but couldn't due to infrastructure deficiencies or deficiencies in the tools themselves. For 
instance, there was extremely poor network connectivity between France and the U.S. Additionally, the application-sharing tool did not support poster- or wall-sized drawings.

Finally, it may be the case that collaboration technologies supporting both synchronous and asynchronous modes might have better supported the CAR engineers. NetMeeting supports synchronous collaboration only. The introduction of asynchronous collaboration technology might have been a useful complement to the synchronous applications, particularly given the small number of overlapping work hours between Europe and the U.S. However, adoption and use of other tools could also have been vulnerable to deficiencies in the available network infrastructure and user inertia.

A second conclusion is that targeted changes in work practices, such as the adoption and use of collaborative tools, need adequate support to ensure success. The required support level varies with the complexity and novelty of the proposed tool. For example, a factor in the widespread adoption of shared, calendar tools within the CAR team was certainly a familiarity with calendars generally, and the seamless transference of knowledge and practices with paper calendars to online, shared calendars. By contrast, for most CAR engineers, NetMeeting represented a completely new tool, with no analogs from past practice. Engineers were asked to master both the tool itself, and the choreography of working with a distant colleague via the tool. The significant investment of one-on-one training surrounding the introduction of NetMeeting was successful for individual users. However, it was not conducted on a broad enough scale to create a critical mass of use for the team in the timeframe of this study.

Less successful was the approach of finding high visibility users to model NetMeeting use, and by example, stimulate wider adoption of the tool. At least within the CAR team, engineers had much to do in performing the normal aspects of their jobs. It was unrealistic to expect that engineers would devote time to instruct colleagues in the use of a sophisticated and complex tool. In addition, some of the users identified as the most influential adopters of new technology had already taken the initiative of finding solutions to their collaboration needs by using an alternative tool. This alternative solution detracted from the effort to broaden the base of NetMeeting users. Although the approach was not as successful as was hoped, at the conclusion of the study, there was evidence within one German site of spontaneous training among engineers beyond the initial, targeted users and diffusion of NetMeeting-use.

A final conclusion is that successful deployment of collaborative tools requires a coordinated approach that involves the IT support organization at an early stage. This project had early endorsement from the IT managers within the CAR team. Also, within Auto 1, the IT managers reported to the human resources organization and the key internal sponsorship came from a human resources vice president. The early endorsement meant that within the constraints of their limited resources, the Auto 1 IT managers were able to smooth many potential barriers to the project. For example, in the course of conducting other business at the European sites, IT personnel checked on the installation of NetMeeting on engineering workstations. IT personnel also installed and maintained an internal NetMeeting server. 


\subsubsection{Advice to researchers [16 (Sec. 4.3.3)]}

The foremost practical recommendation for future research on collaborative tools in field settings would be to anticipate competing demands in this type of research. Specifically, adoption of novel tools with accompanying novel practices is not something that unfolds on a time scale consistent with most projects. Therefore, researchers will confront the need to perform some level of "pump priming." This means there has to be some level of collaboration-tool adoption and use to produce behavior and outcomes that can be used to evaluate the impact of collaborative tools. Realistic determination of resources required to produce use, particularly where none currently exists, is difficult. In this study, for example, UM researchers robustly documented the enthusiastic need for features contained in tools like NetMeeting. Yet, for various reasons, stimulating sufficient levels of use remained a great labor. This labor, in the form of one-on-one training, follow-up visits, and on-going encouragement of use came at the expense of more detailed documentation of what was going on within the CAR team in general, and more specifically, what people were doing with collaborative tools. The compromise solution, including the two survey administrations, was one way to balance the competing demands of tool deployment and training and data gathering. However, as noted earlier, this solution had certain liabilities, such as a poor ability to tease out details of engineering practices and the failure to capture data from all team members.

\subsection{Concluding remarks}

The primary goals of this research were an assessment and better understanding of the adoption, use, impact, and evaluation of collaborative tools in a manufacturing setting. Because the collaborative tool intervention was specifically targeted to needs identified in the requirements gathering phase, a primary research expectation was to find a positive impact of tool use. While there was some indication of improved team performance with use of the tools, the overall adoption did not meet expectations. Although results were mixed, assessment of collaborative tool intervention is often difficult, even at the very coarse granularity of "success" and "failure" [1]. Therefore, one important outcome of this research is the well-documented case study [16], so that over time results can be interpreted more fully, and future research and collaborative tool deployments draw from it.

\section{References}

[1] Blythin, S., J. Hughes, S. Kristoffersen, T. Rodden, and M. Rouncefield. Recognising 'Success' and 'Failure': Evaluating Groupware in a Commercial Context. Proceedings of GROUP'97 (Phoenix, AZ, November 1997), ACM Press, p. 39-46.

[2] Bolon, D. Organizational Citizenship Behavior Among Hospital Employees: A Multidimensional Analysis Job Satisfaction and Organizational Commitment. Journal of Healthcare Management, 42(2), 1997, p. 221-241. 
[3] Box, G., W. Hunter, and J. Hunter. Statistics for Experimenters. John Wiley \& Sons, Inc, New York, 1978.

[4] DeSanctis G. and B. Jackson. Coordination of Information Technology: Team-based Structures and Computer-based Communication Systems. Journal of Management Information Systems, 10, 1994, p. 85-110.

[5] Dix, A., J. Finlay, G. Abowd, and R. Beale, Human Computer Interaction, $2^{\text {nd }}$ Ed., Prentice Hall, Europe, 1998.

[6] Ehrlich, S. Strategies for Encouraging Successful Adoption of Office Communication Systems. ACM Transactions on Information System, 5, 4, 1987, p. 340 - 357.

[7] Finholt, T., E. Rocco, D. Bree, N. Jain, and J.D. Herbsleb. NotMeeting: A Field Trial of NetMeeting in a Geographically-distributed Organization. SIGGROUP Bulletin, 20(1), 1998, p. 66-69.

[8] Glaser, B. and A. Strauss. The Discovery of Grounded Theory: Strategies for Qualitative Research. Aldine, Chicago, 1967.

[9] Glaser, B. and A. Strauss. (1970) Discovery of Substantive Theory: A Basic Strategy Underlying Qualitative Research. In Qualitative Methodology, W. Filstead (Ed.), Rand McNally, Chicago, 1970, p. 288-297.

[10] Katz, D. The Motivational Basis of Organizational Behavior. Behavior Science 9 (2), 1964, p. 131-33.

[11] Mark, G., J. Grudin, and S. Poltrock. Meeting at the Desktop: An Empirical Study of Virtually Collocated Teams. Proceedings of ECSCW'99, Copenhagen, Denmark, 1999, p. 159-178.

[12] Miles, M. and A. Huberman. Qualitative Data Analysis: A Sourcebook of New Methods. Sage Publications, Newbury Park, CA, 1984.

[13] Mohrman, S., J. Galbraith, E. Lawler and Associates. Tomorrow's Organization: Crafting Winning Capabilities in a Dynamic World. Jossey-Bass, San Francisco, CA,1998.

[14] Olson, G., and J. Olson. Distance Matters. Human-Computer Interaction. 15 (2-3), 2000, p.139-178.

[15] Organ, D. Organizational Citizenship Behavior: The Good Soldier Syndrome. Kexington Books, Lexington, MA, 1988. 
[16] Wierba, E. and T. Finholt. A Manufacturing Collaboratory Case Study. National Institute of Standards and Technology. GCR 01-811; 2001 May. (Available at: http://www.nist.gov/msid/collaboratory/doc/gcr-811.pdf) 